\title{
Determinants of Food Taboos in the Pregnant Women of the Awabel District, East Gojjam Zone, Amhara Regional State in Ethiopia
}

\author{
Wollelaw Getnet, ${ }^{1}$ Wubie Aycheh, ${ }^{2}$ and Taddele Tessema $\mathbb{D}^{2}$ \\ ${ }^{1}$ Amhara Regional Health Bureau, Debre Markos Referral Hospital, East Gojjam Zone, Debre Markos, Ethiopia \\ ${ }^{2}$ Department of Public Health, College of Health Science, Debre Markos University, Debre Markos, Ethiopia \\ Correspondence should be addressed to Taddele Tessema; mekutaddele@gmail.com
}

Received 24 January 2018; Accepted 30 July 2018; Published 1 October 2018

Academic Editor: Jennifer L. Freeman

Copyright (C) 2018 Wollelaw Getnet et al. This is an open access article distributed under the Creative Commons Attribution License, which permits unrestricted use, distribution, and reproduction in any medium, provided the original work is properly cited.

Background. Food taboos have great effect on pregnant women through prohibited essential food and/or drinks. It is transferred from generation to generation and has negative effect on pregnant mothers' health. Objective. To assess magnitude of food taboo and associated factors among pregnant women attending antenatal care at public health institutions in Awabel district, Northwest Ethiopia, 2016. Methods. Institutional based cross-sectional study was conducted. Three hundred seven pregnant women were selected for the study. All governmental health institutions in the district were included for the study. Data were entered in to Epi-Data version 3.1 and exported to SPSS version 20 for analysis. Multiple logistic regression analysis was conducted to identify independent predictors of food taboo. Results. Twenty-seven percent of pregnant mother encountered food taboos. Avoided food items by pregnant mothers were linseed, coffee, tea, cabbage, porridge, wheat bread, banana, pimento, groundnut, salty diet, nug, sugarcane, pumpkin, and coca drinks. Reasons mentioned for avoidance of this food items were plastered on the fetal head, making fatty baby which is difficult for delivery, fear of abortion, and fetal abnormality. Age of the mother AOR=2.97 (1.71-5.16), income $\mathrm{AOR}=0.28$ (0.11-0.72), and previous antenatal care $\mathrm{AOR}=2.33$ (1.89-5.47) were significantly associated with food taboo. Conclusion. Our study revealed that considerable proportion of food taboo exists during pregnancy in the study area. This can be improved by strengthening the nutrition counseling components of antenatal care follow-up.

\section{Introduction}

Food taboo is abstaining people from food and/or beverage consuming due to religious and cultural reasons [1]. It can be permanent or temporal. Permanent food taboos are avoiding food and/or drinks throughout their life, while some foods are avoided for certain periods of time. These restrictions often apply to women and are related to the reproduction cycle (during pregnancy, birth, and lactation periods) [2].

Pregnant women have faced dietary deficiency due to food taboo. Some pregnant women, who live in rural area, are obliged to have food taboo that restrain calorie and specific nutrients [3]. Although in the real scenario pregnancy requires more calorie, some food items are considered to be good or bad by the community during pregnancy [4]. Food taboos among pregnant women are varying from culture to culture and community to community especially in rural settings. Pregnant women who were practicing food taboos had significance on lower body weight and unhealthier babies $[5,6]$.

Food taboos have influence on pregnancy even though they need about 300 extra calories per day, especially during the later pregnancy period. When a baby grows quickly, additional calories should come from nutritious foods, so they can contribute to baby's growth and development [7]. The major problem of food taboos is preventing pregnant women from accessing a well-balanced diet, resulting in high prevalence of low birth weight and harm to mother and baby. 
Seven percent of disability particularly sight loss and limb malformation is believed to be caused by broken food taboo [8].

Food taboos have an effect on nutritional status of children and women in Ethiopia. The health sector is better to increase its effort to enhance good nutritional practice through health education, treatment of extremely malnourished children, and provision of micronutrients to mother and children. Government's Health Sector Development Plan IV (HSDP IV) (2010/11-2014/15) continues to improve the nutritional status of pregnant mother and children through the following programmers: Enhanced Outreach Strategy (EOS) with Targeted Supplementary Food (TSF) and Transitioning of EOS into the Health Extension Program (HEP), Health Facility Nutrition Service, Community Based Nutrition $(\mathrm{CBN})$, and Micronutrient Intervention and Essential Nutrition Actions [9].

Any country in the world has food taboo due to different factors like culture, norm, and religion. The food taboo is also differing from place to place and time to time. Each religion has its own food taboo [10]. Food taboos during pregnancy are influenced by different factors like dietary counseling, whether attending antenatal care (ANC) clinic or not, younger age, less educational status, and multiparous and pregnant women. Culture and belief also influence maternal eating pattern during pregnancy [11]. Food taboos on pregnant women usually lead to having low nutritional status and put them at high risk of maternal death. For instance, the mean height of Ethiopian women is $156 \mathrm{~cm}$, which shows severe past malnutrition and puts them at high risk during delivery [5].

Study conducted in Ghana has focused on dietary habit of pregnant women but has not addressed the intake of specific nutrient; especially micronutrient had effect on pregnancy and resulted in complication or not [1]. Consistent evidence supported the fact that Ethiopian women dietary status and habits during pregnancy and nutrient intake were assessed [5]; however, prohibition of food during pregnancy in the study area was not addressed. Maintaining well nutritional status of pregnant women and keeping their health are important by assessing the gap about food taboo. This will give scientific evidence for policy maker and programmers to design possible strategy, to address the problem, furthermore, for the health care workers to intervene based on the finding of the study.

\section{Method and Materials}

2.1. Study Area, Setting, and Period. The study was conducted in Awabel district, East Gojjam Zone, Amhara Region, Ethiopia. Awabel district is $40 \mathrm{~km}$ from Debre Markos in the Southeast and $259 \mathrm{~km}$ from Addis Ababa in the Northwest and $306 \mathrm{~km}$ from Bahir Dar in the Southwest. The district has 5 urban and 15 rural kebeles, six health centers, and one district hospital. It has 137,000 total population. The study was conducted from April 15 to May 17, 2016.

2.2. Study Design and Population. Institutional based crosssectional study was conducted among women attending ANC follow-up from all governmental heath institutions in the district.

2.3. Sample Size and Sampling Technique. The sample size determination was used with the assumption of confidence level $=95 \%$, critical value $Z=1.96$ (from significance level $\alpha=5 \%$ ), and degree of precision $=0.05$, by taking $49.8 \%$ proportion from Shashmene district study about food taboo [5]. Then the sample size was calculated using the formula for single population proportion.

$\mathrm{n}_{0}=\left(\mathrm{S}_{\mathrm{o} / 2}\right)^{2}$. P $(1-\mathrm{p}) / \mathrm{W}^{2}=(1.96)^{2} . \quad(0.498) . \quad(0.502) /$ $(0.05)^{2}=384$, where $n_{o}$ is sample size calculated as follows. Since the target population in the study area was less than 10,000 (i.e., 1250), we use the formula $n=n_{0} / 1+n_{o} / N$, where $\mathrm{N}$ is the number of pregnant women:

$=384 /(1+384 / 1250)=293$.

Then we can take/add $5 \%$ for nonresponse rate $293 * 5$ / $100=14$.

So, total sample size was 307 .

2.4. Sampling Procedure. Based on the previous ANC followup number, the sample was allocated for each health institution. So from each of the six health centers we should expect to collect 37 samples and from the primary hospital 82 samples. Sequential sampling technique was used to select study participants. According to the sample size in each health institution samples were taken.

2.5. Data Collection Methods. An interview administered pretested Amharic language questionnaire prepared in English was used. To avoid information contamination, pretest was done on 5\% women attending ANC follow-up on the near health center and its finding was used to modify the tool. Data collectors were briefed about the purpose of the study and data were collected after a verbal informed consent obtained from participants. Data collection process was facilitated by seven midwifery professional data collector and two BSc midwives.

2.6. Data Quality Assurance. Data quality was assured through careful design of the questionnaire. Data collector and supervisors were trained one day about the purpose of the study, the questionnaire in detail, the data collection procedure, the data collection setting, and the rights of study participants. Pretest was done prior to the actual data collection.

2.7. Data Processing and Analysis. Data were checked for completeness and consistency and entered in to Epi-Data version 3.1 and then exported to SPSS version 20 for analysis. Descriptive analysis was carried out to determine the magnitude of food taboo. Bivariate logistic regression analysis which was performed for each independent variable with the outcome of interest at $\mathrm{p}<0.05$ was considered statistically significant. Finally multiple logistic regression analysis was conducted to determine independent predictors of food taboo. 
TABLE 1: Sociodemographic characteristics of ANC follow-up women in Awabel district, Northwest Ethiopia, April 15-May 17, 2016 (n=307).

\begin{tabular}{|c|c|c|c|}
\hline Variables & & Frequency & Percentage \\
\hline \multirow[t]{4}{*}{ Age group } & $15-19$ years & 49 & 16.3 \\
\hline & 20-24 years & 134 & 44.7 \\
\hline & $25-29$ years & 93 & 31.0 \\
\hline & $\geq 30$ years & 24 & 8.0 \\
\hline \multirow[t]{2}{*}{ Religion } & Orthodox & 261 & 87.0 \\
\hline & Muslim & 39 & 13.0 \\
\hline \multirow[t]{2}{*}{ Ethinicity } & Amhara & 273 & 91.0 \\
\hline & Oromo & 27 & 9.0 \\
\hline \multirow{5}{*}{ Educationl Status } & Cannot read and write & 141 & 47.0 \\
\hline & Can read and write & 68 & 22.7 \\
\hline & Primary school & 15 & 5.0 \\
\hline & secondary school & 33 & 11.0 \\
\hline & College diploma and above & 43 & 14.3 \\
\hline \multirow[t]{4}{*}{ Occupation mother } & Farmer & 170 & 56.7 \\
\hline & Government employee & 46 & 15.3 \\
\hline & Merchant & 55 & 18.3 \\
\hline & Housewife & 29 & 9.7 \\
\hline \multirow[t]{2}{*}{ Marital status } & Married & 289 & 96.3 \\
\hline & Unmarried & 11 & 3.7 \\
\hline \multirow[t]{4}{*}{ Number of deliver } & Primi & 161 & 53.7 \\
\hline & Two times & 80 & 26.7 \\
\hline & Three times & 40 & 13.3 \\
\hline & $=/>4$ times & 19 & 6.3 \\
\hline \multirow[t]{4}{*}{ Income } & $<650$ birr & 140 & 46.7 \\
\hline & 651-1000birr & 64 & 21.3 \\
\hline & 1001-1500birr & 44 & 14.7 \\
\hline & $\geq 1500$ birr & 52 & 17.3 \\
\hline \multirow[t]{4}{*}{ Educational status of husband } & Cannot read \&write & 147 & 49.0 \\
\hline & Can read and write & 87 & 29.0 \\
\hline & Complete primary and secondary school & 26 & 8.6 \\
\hline & College diploma \& above & 40 & 13.4 \\
\hline \multirow[t]{2}{*}{ Previous ANC attendance } & Yes & 224 & 74.7 \\
\hline & No & 76 & 25.3 \\
\hline \multirow[t]{2}{*}{ Residence } & Rural & 239 & 79.7 \\
\hline & Urban & 61 & 20.3 \\
\hline
\end{tabular}

2.8. Ethical Consideration. The study was conducted after securing approval of proposal by Debre Markos University, Medicine and Health Sciences College Ethical Review Committee. Written permission was obtained from Awabel Administrative Health Office for Selected Health Centers. All the study participants were informed about the purpose of the study and finally verbal consent was obtained before interview. The study participants have the right to refuse participation or terminate their involvement at any point when the interview was secured.

\section{Results}

3.1. Sociodemographic Characteristics. Among 307 respondents, data were collected from 300 pregnant women; this made the response rate $97.7 \%$.

About $87 \%$ of the respondents were orthodox Christian and $91 \%$ were from Amhara ethnicity. Around 50\% of the pregnant women were illiterate and $79.4 \%$ of the pregnant women lived in rural areas (Table 1).
3.2. Food Taboos during Pregnancy. Twenty-seven percent of respondent avoid three or more food/drink items during pregnancy. Twelve food and/or drink items were prohibited by the study participants.

3.2.1. Fruits and Vegetables. Certain fruit and vegetables were taboo during pregnancy such as banana 107 (35.7\%), pimento $96(32 \%)$, cabbage $73(24.3 \%)$, and sugarcane $133(44.3 \%)$. Pregnant women believed that the reason for the taboo is that when they consumed banana, something is attached to the head of the fetus, pimento burns the fetus, cabbage disturbs the fetus, and sugarcane increases the seminal fluids (Figure 1).

3.2.2. Cereals and Salty Diet. The result of this study indicated that cereals were taboos like linseed (16.3\%), pumpkin (42.7\%), nug (32.3\%), wheat (28.3\%), groundnut (13.7\%), and salty diets (11. 7\%). The study participants avoid pumpkin and ground nut because they assumed that these foods increase 
TABLE 2: Determinants of food taboo in public health institution in Awabel district, Northwest Ethiopia, April 15-May 17, 2016 (n=307).

\begin{tabular}{|c|c|c|c|c|c|c|}
\hline \multirow{2}{*}{ Variables } & & \multicolumn{2}{|c|}{ Having food taboo } & \multirow{2}{*}{$\operatorname{COR}(95 \% \mathrm{CI})$} & \multirow{2}{*}{ AOR (95\%CI) } & \multirow{2}{*}{ P-Value } \\
\hline & & Yes & No & & & \\
\hline \multirow{4}{*}{ Age of the mother } & $15-19$ years & 11 & 38 & 1.00 & 1.00 & \\
\hline & $20-24$ years & 40 & 94 & $0.68(0.316,0.464)$ & 2.971(1.711,5.159) & 0.001 \\
\hline & $25-29$ years & 28 & 65 & $0.672(0.301,0.502)$ & $3.358(1.638,6.886)$ & 0.001 \\
\hline & $\geq 30$ years & 2 & 22 & $3.18(1.646,15.70)$ & $12.716(2.429,66.58)$ & 0.003 \\
\hline \multirow{4}{*}{ Income of the mother } & $<650$ biir & 21 & 119 & 1.00 & 1.00 & \\
\hline & 650-1000birr & 22 & 42 & $0.337(0.168,0.674)$ & $0.290(0.132,0.638)$ & 0.002 \\
\hline & 1001-1500birr & 16 & 28 & $0.309(0.143,0.667)$ & $0.281(0.111,0.715)$ & 0.008 \\
\hline & $\geq 1500$ birr & 22 & 30 & $0.241(0.117,0.494)$ & $0.330(0.117,0.929)$ & 0.036 \\
\hline \multirow{2}{*}{$\begin{array}{l}\text { Previous ANC } \\
\text { attendants }\end{array}$} & Yes & 63 & 18 & 1.00 & 1.00 & \\
\hline & No & 161 & 58 & $1.22(1.89,5.408)$ & $2.33(1.257,4.336)$ & 0.007 \\
\hline
\end{tabular}

Note: COR: crude odds ratio; AOR: adjusted odds ratio; CI: confidence interval.

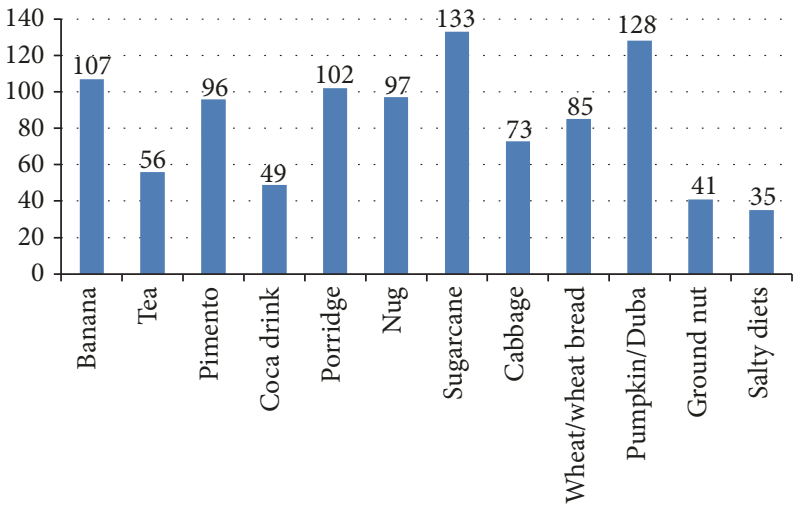

Y-axis Number of pregnant women involved in food taboo

$\mathbf{X}$-axis List of food items

Figure 1: Food taboo in ANC attendants in each food items/drinks in Awabel district, Northwest, Ethiopia, April 15-May 17, 2016.

the weight of the fetus, making it difficult to deliver, linseed causes loss strength of the fetus, and nug changes the color of the fetus and makes it black.

3.2.3. Drinks. Drinks like coffee (19\%), tea (18.7\%), coca $(16.3 \%)$, and porridge (34\%) were restricted due to burning the fetus and causing abnormality and coca drink causes abortion.

These foods were restricted by the following reasons: banana (believing that something is attached to the head), pimento (burning the fetus), cabbage (disturbing the fetus), and sugarcane (increasing the fluids during delivery); coffee and tea were restricted due to burning the fetus and causing abnormality; coca drink causes abortion; pumpkin and ground nut increase the weight of the fetus making it difficult to deliver. Most of pregnant women avoid foods due to plaster on the fetal head, making fatty baby, difficult for delivery, fear of abortion, and fetal abnormality.

3.3. Determinants of Food Taboos in the Pregnant Women. Pregnant women whose age is 20-24 years were 2.97 times more likely to develop food taboo compared with the age between 15 and 19 years $(\mathrm{AOR}=2.97,95 \% \mathrm{CI}: 1.71-5.16)$; pregnant women who earned 650-1000 birr are 56\% less likely to have food taboo compared to pregnant women who have $<650$-birr monthly income (AOR $=0.29,95 \% \mathrm{CI}$ : 0.13-0.64); and pregnant women who never have ANC attendance in the health institution were 2.33 times more likely to develop food taboo as compared with pregnant women who have ANC attendance $(\mathrm{AOR}=2.3,95 \% \mathrm{CI}: 1.26-4.34)$ (Table 2$)$.

\section{Discussion}

The finding of this study revealed that $27 \%$ of study participants had food taboo which is less than the proportion of food taboo in different studies done, in Shashmene district (49.8\%) [4].

More than three-fourths $(82.1 \%)$ of participants in North Costal Paradesh and nearly half (48\%) of participants in Surendranagar had food taboo which are higher than our findings $[5,6]$.

The possible difference may be because of study time, study area, and increase in the knowledge of the mother in the time of the study. In this study, some fruits were avoided even though they are important in the period of pregnancy. Based on this study, banana has not been eaten by 107(35.7\%) of pregnant women which is not comparable with the study conducted in Hydya Zone (8.6\%). This discrepancy may be due to the study area, culture, and time difference [12].

Ground nut in this study was $13.7 \%$ which is in line with the study done in Shashmene (13.6\%) [4]. Age of the mother in bivariate regression analysis was significantly associated with food taboos; as age of the mother is increased, adoption of the food taboo is increased. Pregnant women whose age is 20-24 years were 6.8 times more likely to develop food taboos compared with age between 15 and 19 years $(\mathrm{COR}=8.6,95 \%$ CI: 0.32-0.46)

In multivariate regression analysis, age of the mother was significantly associated with food taboo. As the age of the mother is increased, adoption of the food taboo is increased. Pregnant women whose age is $20-24$ years were 2.97 times 
more likely to develop food taboos compared with the age between 15 and 19 years ( $\mathrm{AOR}=2.97,95 \% \mathrm{CI}$ : $1.71-5.16)$. This study is almost similar to the study in Shashmene [4]. The possible explanation may be that younger women more likely accept modern education and older women have indigenous knowledge.

Previous ANC attendance of study participant is significantly associated with food taboo. Pregnant women who have never had ANC attendance in the health institution were 2.33 times more likely to develop food taboo as compared with pregnant women who have had ANC attendance. This result is comparable with the study conducted in Shashmene [4]. This may be due to the knowledge gained from formal education and experienced health education. In this study, income was significantly associated with food taboo. Pregnant women who earned 650-1000 birr were 56\% less likely to have food taboo compared to pregnant women who have $<650$-birr monthly income, which is in line with the study done in Wondo Genet [12], Bangladesh [13], and Ogan state of Nigeria [14]. As income increases, the avoidance of food taboos decreases.

\section{Conclusion}

There were low proportion of food taboos in the study area and were obligated to avoid specific food items due to cultural and traditional view. Women, who were of old age, had low income, and had not had previous ANC attendance, were more practicing food taboos.

The food and drink items, which were avoided during pregnancy, were linseed, cabbage, banana, sugarcane, pumpkin/duba, nug, tea, coffee, porridge, coca drink, groundnut, pimento, and salty diets. Reasons which avoid food were plastered on the fetal head, fatty baby, fear of abortion, and fetal abnormality. Age of the mother, income, and previous ANC attendance had significant association with food taboos.

Based on the finding, the following recommendations were forwarded:

(I) Midwives had better work more for creating awareness about food taboos. Women, who had food taboos, assess the reasons and provide health education about the use of appropriate nutrition.

(II) Governmental, nongovernmental organization and various public associations had been better actively involved in eliminating harmful beliefs.

(III) Health education program had better taken cognizance of the popular beliefs regarding food taboos during pregnancy and used innovative means to minimize their negative and maximize their positive nutritional effects.

(IV) The health professionals, who work in Awabel District Health Institutions, had better created awareness for pregnant women who have ANC follow-up to minimize food taboos.

\section{Data Availability}

The data used to support the findings of this study are available from the corresponding author upon request.

\section{Conflicts of Interest}

The authors declare that they have no conflicts of interest.

\section{Authors' Contributions}

Wollelaw Getnet, Wubie Aycheh, and Taddele Tessema were participated in proposal writing, analyzing the data, and drafting the paper. Taddele Tessema prepared the manuscript for publication. All authors revised subsequent drafts of the paper.

\section{Acknowledgments}

The authors' deep gratitude goes to Debre Markos University, College of Medicine and Health Sciences, for proper review and approval of this paper. The authors would also like to extend their gratitude to Awabel district health workers, data collectors, and supervisors for valuable contribution for the success of this study.

\section{References}

[1] "Harmful traditional practices affecting the health of women and children," Factsheet No 23, vol. 10, 1997.

[2] O. Esther, Relation of dietary and socio economic characteristics of mothers to child growth [Master thesis], 2008.

[3] P. Rajkumar and M. A. Vedapriya, "Taboos and misconceptions about food during pregnancy among rural population of pondichery," Calicut Medical Journal, vol. 8, no. 2, p. 1, 2010.

[4] N. Biza Zepro, "Food Taboos and Misconceptions Among Pregnant Women of Shashemene District, Ethiopia, 2012," Science Journal of Public Health, vol. 3, no. 3, p. 410, 2015.

[5] A. Parmar and G. H. Khanpara, "A study on taboos and misconceptions associated with pregnancy among rural women of Surendranagar district," Healthline, vol. 2, p. 40, 2013.

[6] Lakshmi, "Food preference and taboos during ANC among the tribal women," Journal of community nutrition and health, vol. 2, no. 2, p. 33, 2013.

[7] K. A. Samson, "Common food taboos and beliefs during pregnancy in Yilokrobo district, Ghana," Science Journal of Public Health, vol. 2, no. 3, p. 41, 2014.

[8] J. DelmaPaofa, N. Kaugla, T. Catherina et al., "Food taboos and traditional customs among pregnant women in Papua New Guinea: Missed opportunity for education in antenatal clinics," Research Journal reproductive health, vol. 8, no. 1, p. 6, 2013.

[9] Central Statistical Agency, Ethiopia Mini Demographic and Health Survey, vol. 56, Addis Ababa, Ethiopia, 2014.

[10] J. T. Liu, “Taboos in Food Practices during Pre and Post-natal: A Comparative Study between Tribal and Non- Tribal Women in Odisha," Journal of reproductive health, vol. 2, no. 3, pp. 141-152, 2015.

[11] O. A. Oni and J. Tukur, "Identifying pregnant women who would adhere to food taboos in a rural community: a community-based study." African Journal of Reproductive Health, vol. 16, no. 3, pp. 68-76, 2012.

[12] D. Kuche, P. Singh, and D. Moges, "Dietary Practices And Associated Factors among Pregnant Women in Wondo Genet District, Southern Ethiopia: A Cross-Sectional Study," Journal 
of Pharmaceutical \& Scientific Innovation, vol. 4, no. 5, pp. 270275, 2015.

[13] H. B, "Nutritional Status of Pregnant Women in Selected Rural and Urban Area of Bangladesh," Journal of Nutrition \& Food Sciences, vol. 03, no. 04, 2013.

[14] O. S. Oluwafolahan, A. B. Catherine, and A. J. Olubukunola, "Dietary habits of pregnant women in Ogun-East Senatorial Zone, Ogun State, Nigeria: A comparative study," International Journal of Nutrition and Metabolism, vol. 6, no. 4, pp. 42-49, 2014. 


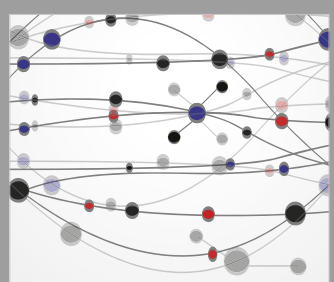

The Scientific World Journal
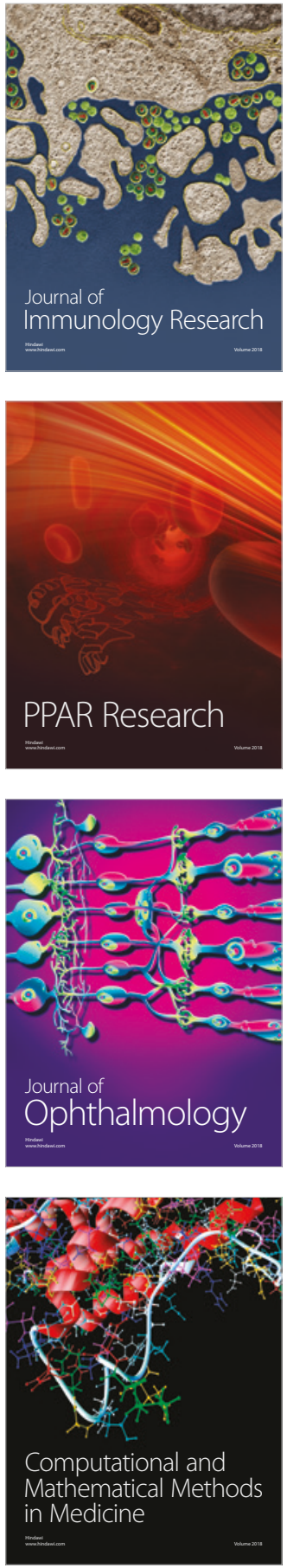

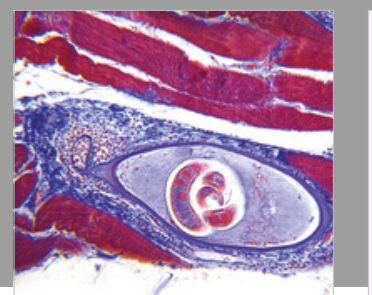

Gastroenterology Research and Practice

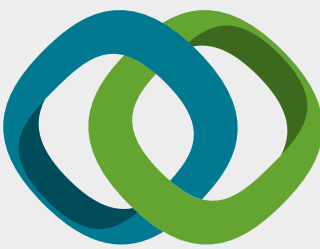

\section{Hindawi}

Submit your manuscripts at

www.hindawi.com
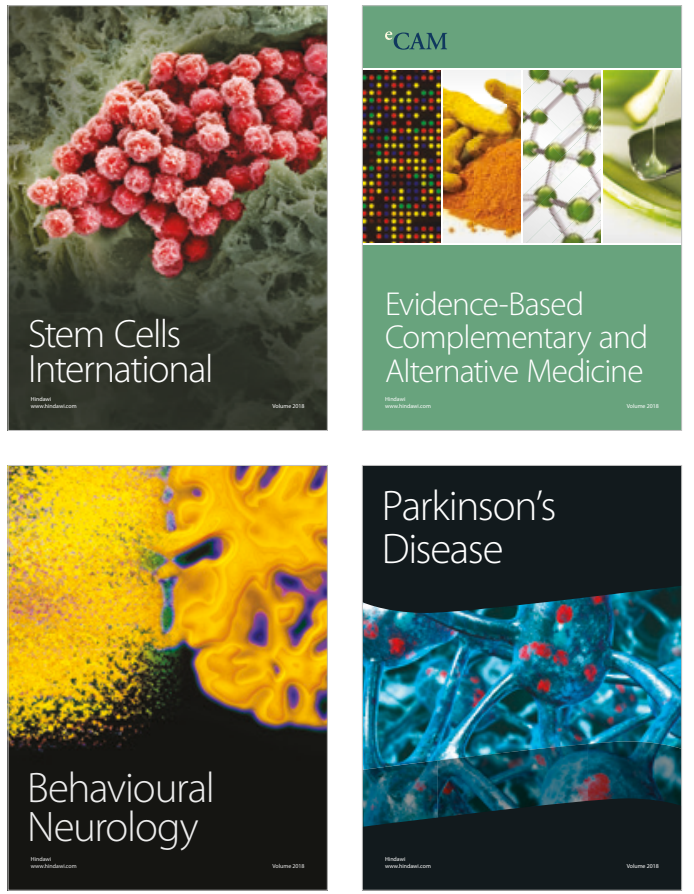

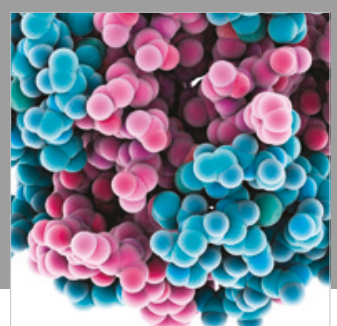

ournal of

Diabetes Research

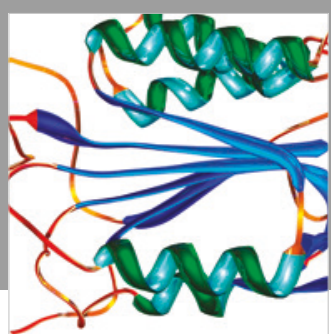

Disease Markers
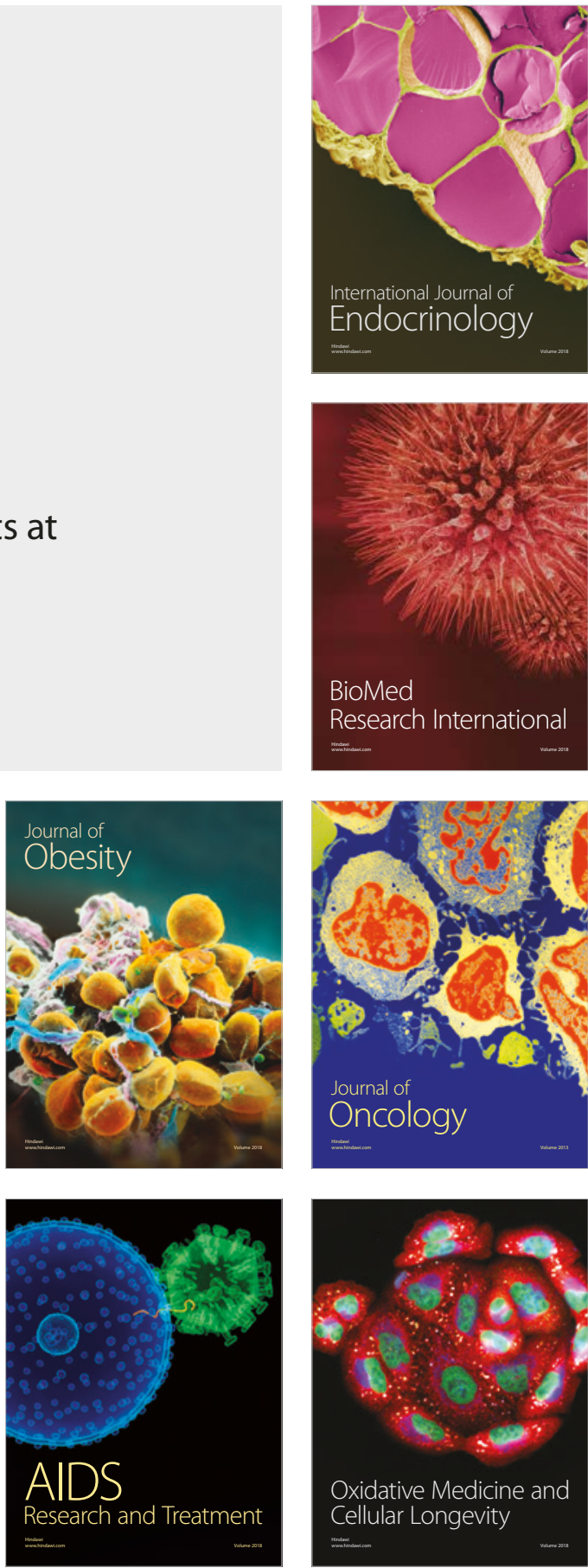